\title{
Dark-bright exciton coupling in asymmetric quantum dots
}

\author{
Savvas Germanis, ${ }^{1, *}$ Paola Atkinson, ${ }^{1}$ Richard Hostein, ${ }^{1}$ Catherine Gourdon, ${ }^{1}$ Valia Voliotis,,${ }^{1}$ Aristide Lemaitre, ${ }^{2}$ \\ Mathieu Bernard, ${ }^{1}$ Florent Margaillan, ${ }^{1}$ Silbé Majrab, ${ }^{1}$ and Benoit Eble ${ }^{1, \dagger}$ \\ ${ }^{1}$ Sorbonne Universités, UPMC Université Paris 06, CNRS, Institut des Nanosciences de Paris, 4 place Jussieu,75252 Paris, France \\ ${ }^{2}$ Centre de Nanosciences et de Nanotechnologies, CNRS, Université Paris-Sud, Université Paris-Saclay, 91460 Marcoussis, France
}

(Received 16 February 2018; published 5 October 2018)

\begin{abstract}
Symmetry breaking of the confinement in $\operatorname{In}(\mathrm{Ga})$ As quantum dots leads to mixing between the bright and dark exciton states and an increase in the oscillator strength of the dark exciton. We demonstrate here that the change in degree of linear polarization of the bright and dark excitons as a function of applied magnetic field, where both parallel and perpendicular magnetic field components are applied, can be used to quantify the dark-bright coupling. Experimental results for a dot where the coupling is weak and the dark exciton is undetectable in zero applied magnetic field are in good agreement with theoretical simulations based on atomistic calculations reported by M. Zieliński et al. [Phys. Rev. B 91, 085403 (2015)].
\end{abstract}

DOI: 10.1103/PhysRevB.98.155303

\section{INTRODUCTION}

The ability to transfer quantum information between a photon and a single spin make semiconductor quantum dots (QDs) ideal for the solid-state implementation of quantum information and communication protocols [1-3]. Although resonant excitation of the so-called bright exciton (BE), corresponding to an electron-hole pair with antiparallel spins $\left(J_{z}= \pm 1\right)$, has led to high-fidelity on-demand generation of indistinguishable photons [4] and coherent manipulation of single-electron spins confined in a $\mathrm{QD}$, the $\mathrm{BE}$ coherence time remains limited to a few hundreds of picoseconds [5].

In contrast, the dark exciton (DE), corresponding to an electron-hole pair with parallel spins $\left(J_{z}= \pm 2\right)$, has been shown to have coherence times of the order of 5-100 ns [6,7]. This makes it a highly attractive candidate for a qubit if the DE can be made optically active.

The brightening of the DE, opening the way to an alloptical manipulation scheme, is theoretically predicted to occur by symmetry breaking of the QD potential $[6,8]$. Atomistic calculations reported in Ref. [9] show that a weak-symmetry reduction in the plane containing the growth axis, lowering the QD point group symmetry from $C_{2 v}$ to $C_{s}$, plays a major role in the DE optical activity [10]. For this purpose, truncated lens-shaped InGaAs QDs were used to calculate the dark-bright (DB) exciton coupling strength. The calculated coupling strength was found to be weak, on the order of a few $\mu \mathrm{eV}$.

Brightening of the DE through coupling with the $\mathrm{BE}$ should lead, in principle, to a shortening of the coherence time. However, it is worth noting that results presented in Ref. [7] still demonstrate a DE coherence time as long as $100 \mathrm{~ns}$, while the DE to BE photoluminescence (PL) ratio was as strong as 1:3 in the weak-excitation regime.
The DE has previously been studied by a variety of techniques. Nonresonant excitation allows the spin dynamics of capture and decay of the DE to be inferred [9,11-13]. Alternatively, applying a transverse magnetic field (Voigt geometry) leads to a mixing between the bright and dark states, resulting in the appearance of two DE branches in addition to the two $\mathrm{BE}$ branches in magnetoluminescence spectra. These spectra allow the determination of the DB splitting $\Delta_{D B}$ and the DE fine-structure splitting $\Delta_{2}$ [14] (Fig. 1). In CdTe/ZnTe QDs, an anticrossing between the lower BE and the upper DE branches has been seen when the DB coupling is of the same order of magnitude as the DB splitting $[15,16]$. However, in InGaAs dots, an apparent crossing between the branches is typically observed, indicating that DB coupling is probably very weak.

Here, we propose an experimental method to measure such a weak DB coupling. In Sec. II, we introduce a heuristic theoretical approach describing the symmetry-breakinginduced valence band mixing (VBM) responsible for the DB coupling. Then in Sec. III, the experimental results and the method used to quantify this coupling are presented. This method is based on the analysis of the DE linear polarization with a slightly tilted magnetic field relative to the Voigt configuration.

\section{VALENCE-BAND-MIXING-INDUCED DARK-BRIGHT COUPLING}

\section{A. Enhancement of valence band mixing}

It is well established that the valence band is very sensitive to the reduced QD symmetry, which alters the pure heavyhole $(\mathrm{HH})$ character [17-19] and mixes the HH with the light hole state $(\mathrm{LH})$ states. We can write the hole states as a linear combination of $\mathrm{HH}$ and $\mathrm{LH}$ as

$$
\begin{aligned}
& \left\langle\mathbf{r} \mid \Psi_{h}^{\uparrow}\right\rangle \sim \chi_{h h}(\boldsymbol{r})|3 / 2,+3 / 2\rangle+\chi_{l h}(\boldsymbol{r}) \\
& \times[\beta|3 / 2,-1 / 2\rangle+\gamma|3 / 2,+1 / 2\rangle]
\end{aligned}
$$

*savvas.germanis@insp.jussieu.fr

†benoit.eble@insp.jussieu.fr 

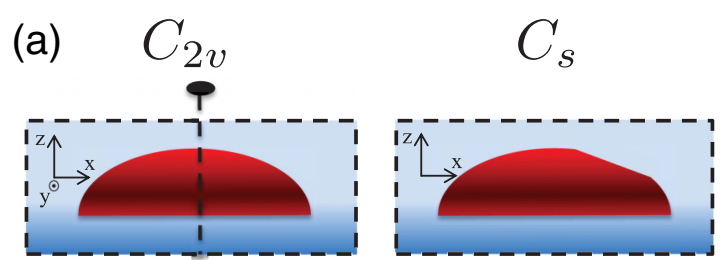

(b)

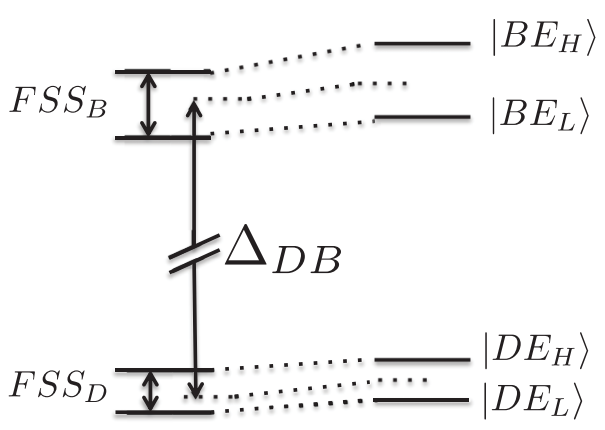

FIG. 1. (a) Schematic QD representations of different point group symmetries, $C_{2 v}$ on the left with the twofold symmetry axis and the first mirror plane $(x z)$ and $C_{s}$ on the right with the only mirror plane ( $x z$ ). (b) Energy level in $C_{2 v}$ and $C_{s}$ symmetries. We used the condition $\Delta_{11} \Delta_{12}<0$, implying a positive energy shift of the level energies in the $C_{s}$ symmetry compared to $C_{2 v}$.

and

$$
\begin{aligned}
\left\langle\mathbf{r} \mid \Psi_{h}^{\downarrow}\right\rangle \sim & \chi_{h h}(\boldsymbol{r})|3 / 2,-3 / 2\rangle+\chi_{l h}(\boldsymbol{r}) \\
& \times\left[\beta^{*}|3 / 2,+1 / 2\rangle-\gamma^{*}|3 / 2,-1 / 2\rangle\right],
\end{aligned}
$$

where $\left|J, J_{z}\right\rangle$ correspond to the periodic part of the Bloch wave function and $\chi_{h h(l h)}$ are the envelope wave functions associated with the $\mathrm{HH}$ and LH states.

The VBM leads to a change in the polarization properties of the QD luminescence. The mixing of $|3 / 2, \pm 3 / 2\rangle$ with $|3 / 2, \mp 1 / 2\rangle$ states, through the parameter $\beta$, controls the anisotropy of the BE polarization diagram along the growth direction [20-22] and confers a $z$-dipole component to the $\mathrm{DE}$ which allows the DE to be detected perpendicular to the growth direction $[23,24]$. The mixing of $|3 / 2, \pm 3 / 2\rangle$ with $|3 / 2, \pm 1 / 2\rangle$, (i.e., $\gamma$ ) does not affect the in-plane polarization, only the polarization of BE luminescence perpendicular to the growth direction [20]. That is why, in most cases, $\gamma$ does not need to be considered when analyzing the PL along the growth direction. However, it plays a major role in the DB coupling.

For this reason, we show here how a reduction in QD symmetry significantly enhanced $|\gamma|$. For this purpose, two different shapes of QDs are considered as typical models associated with the $C_{2 v}$ and $C_{s}$ point group symmetries [see Fig. 1(a)]: (i) a lens with an elliptical base and, following Ref. [10], (ii) an ellipsoidal lens truncated by an oblique facet on top of the QD.

One can derive the VBM parameters in the framework of the four-band model, for which the notations are given in Appendix A.
Using degenerate-state perturbation theory, VBM parameters can be expressed by

$$
\begin{aligned}
& \beta=\frac{e^{i 2 \psi} 2 w_{\|}}{1+\sqrt{1+4 w_{\|}^{2}+4 w_{\perp}^{2}}}, \\
& \gamma=\frac{e^{i 2 \Theta} 2 w_{\perp}}{1+\sqrt{1+4 w_{\|}^{2}+4 w_{\perp}^{2}}} .
\end{aligned}
$$

We have used the following set of definitions: $w_{\|}=$ $\frac{\left|\left\langle\chi_{h h}\left|R_{k}+R_{\epsilon}\right| \chi_{l h}\right\rangle\right|}{\Delta_{l h}}$ and $w_{\perp}=\frac{\left|\left\langle\chi_{h h}\left|S_{k}+S_{\epsilon}\right| \chi_{l h}\right\rangle\right|}{\Delta_{l h}}$ are the moduli of the $\mathrm{HH}$ to LH couplings, where $R_{k / \epsilon}$ and $S_{k / \epsilon}$ are defined in Appendix A and $2 \psi$ and $2 \Theta$ their respective arguments.

From Eqs. (3) and (4), it can be seen that VBM has at least two origins: anisotropy of the heterostructure potential and the anisotropic strain field natively present in self-assembled QDs.

For the two different QD shapes being considered, $\beta$ is expected to deviate significantly from zero as $\beta$ is determined by the QD anisotropy perpendicular to the growth direction. This is shown by the fact that $w_{\|}$is not equal to zero due to the presence of biaxial strain [i.e., $\left\langle\chi_{h h}\left|\epsilon_{x x / y y}(\mathbf{r})\right| \chi_{l h}\right\rangle \neq 0$ ] and to the asymmetry between the $x$ and $y$ direction imposed by the ellipsoidal shape of the $\operatorname{dot}$ (i.e., $\left\langle\chi_{h h}\left|k_{x}^{2}-k_{y}^{2}\right| \chi_{l h}\right\rangle \neq 0$ ). $|\beta|$ is typically in the range of 0.1 to 0.7 (see, for example Ref. [25]), when the dominant contribution is given by the strain [19].

The facet introduced to break the $C_{2 v}$ symmetry leads to two important modifications: (i) the heterostructure potential no longer has two mirror planes ( $x y$ and $y z$ ) but now has only one mirror plane $(x z)$ and (ii) the disruption at the interface between the QD and the GaAs semiconductor matrix introduced here by the facet leads to a huge shear strain within the QD [26]. Thus, $w_{\perp}$ which is proportional to $\left\langle\chi_{l h}\right| k_{x} k_{z}-$ $i k_{y} k_{z}\left|\chi_{h h}\right\rangle$ is no longer zero since, due to the QD facet, the $\mathrm{HH}$ and $\mathrm{LH}$ envelope wave functions also no longer have two mirror plane symmetries [27] due to modification (i). In the same way, the additional strain contribution induced by the facet in modification (ii) also leads to nonzero $|\gamma|$ as $\left\langle\chi_{h h}\left|S_{\epsilon}\right| \chi_{l h}\right\rangle \propto\left\langle\chi_{h h}\left|\epsilon_{x z}(\mathbf{r})\right| \chi_{l h}\right\rangle \neq 0$. As opposed to $\beta, \gamma$ originates from any deformation which develops along the growth axis, and hence, the loss of the rotational invariance of the $C_{s}$ symmetry increases $|\gamma|$.

\section{B. Role of the electron/hole exchange interaction}

The influence of the VBM on the exciton fine-structure splitting (FSS) requires us to evaluate the electron/hole exchange matrix elements [28-32], which are split into two contributions: short and long range. The short-range contribution takes the effective Hamiltonian form [16]

$$
\hat{H}_{\mathrm{exc}}^{s r}=-\sum_{i=x, y, z} \frac{2}{3} \delta_{0}^{s r} \hat{\sigma}_{i}^{e} \hat{J}_{i}^{h}+b_{i} \hat{\sigma}_{i}^{e}\left(\hat{J}_{i}^{h}\right)^{3},
$$

where $\hat{\sigma}_{i}^{e}$ and $\hat{J}_{i}^{h}$ are the spin operators associated with the electron and hole, respectively. The magnitude of the linear term dominates over the cubic term, i.e., $\delta_{0}^{s r}>b_{i}$ [30]. The exchange terms are recast in the matrix form using the natural exciton basis $|\widetilde{ \pm 1}\rangle=\left|\Psi_{e}^{\downarrow(\uparrow)}\right\rangle \otimes\left|\Psi_{h}^{\uparrow(\downarrow)}\right\rangle$ and $|\widetilde{ \pm 2}\rangle=$ $\left|\Psi_{e}^{\uparrow(\downarrow)}\right\rangle \otimes\left|\Psi_{h}^{\uparrow(\downarrow)}\right\rangle$, with $\left\langle\boldsymbol{r} \mid \Psi_{e}^{\uparrow(\downarrow)}\right\rangle=\chi_{e}(\boldsymbol{r})|1 / 2, \pm 1 / 2\rangle$ being 
the conduction electron state:

$$
\hat{H}_{\mathrm{exc}}=\frac{1}{2}\left[\begin{array}{cccc}
\Delta_{0} & \Delta_{1} e^{2 i \theta} & \Delta_{11} & \Delta_{12} \\
\Delta_{1} e^{-2 i \theta} & \Delta_{0} & -\Delta_{12} & -\Delta_{11} \\
\Delta_{11}^{\star} & -\Delta_{12}^{\star} & -\Delta_{0} & \Delta_{2} \\
\Delta_{12}^{\star} & -\Delta_{11}^{\star} & \Delta_{2} & -\Delta_{0}
\end{array}\right],
$$

with

$$
\begin{gathered}
\Delta_{0}=\delta_{0}^{s r}+\delta_{0}^{l r}, \\
\Delta_{1} e^{2 i \theta}=\frac{3}{4}\left(b_{y}-b_{x}\right)-\frac{4}{\sqrt{3}} \beta^{*} \alpha_{e, l h} \delta_{0}^{s r}+\delta_{1}^{l r}, \\
\Delta_{2}=-\frac{3}{4}\left(b_{y}+b_{x}\right), \\
\Delta_{11}=-\frac{2}{3} \sqrt{3} \gamma \alpha_{e, l h} \delta_{0}^{s r}+\delta_{11}^{l r}, \\
\Delta_{12}=\frac{2}{3} \beta^{*} \gamma^{*} \alpha_{e, 2 l h} \delta_{0}^{s r}+\delta_{12}^{l r} .
\end{gathered}
$$

In the above expressions, the short-range contributions are calculated explicitly as a function of $\delta_{0}^{s r}$, VBM parameters, and electron-hole overlaps $\alpha_{e, l h}$ and $\alpha_{e, 2 l h}$ coming from the normalization procedure (see Appendix B). The phase factor $e^{2 i \theta}$ in Eq. (8) refers to the angle $\theta$ between the QD main elongation axis and the [110] crystallographic axis [20,33]. The calculation of the long-range part labeled $\delta_{i}^{l r}$, with $i \in$ $\{0,1,11,12\}$, is beyond the scope of this work, as it requires sophisticated methods such as multipole expansion of the Coulomb integrals [34]. So far, the effect of the VBM on the long-range electron/hole exchange interaction has been studied theoretically for the case of the BE FSS [35], showing corrections proportional to the VBM parameters and to $\delta_{0}^{l r}$. However, the DB coupling was not considered in this work. When $\Delta_{11}, \Delta_{12}=0$, i.e., $\gamma=0$, the electron/hole exchange Hamiltonian $\hat{H}_{\text {exc }}$ reduces to the well-known exchange interaction in $C_{2 v}$ symmetry [16] $\hat{H}_{C_{2 v}}$.

Our approach generalizes the calculations presented in Refs. [28,29] by taking into account all the LH components and indicates that the $\gamma$ parameter is directly responsible for the mixing $\Delta_{11}$ between the $|\widetilde{ \pm 1}\rangle$ and $|\widetilde{ \pm 2}\rangle$ states through the linear term [31] in Eq. (5). Moreover, this $\gamma$ parameter can potentially be tuned by applying strain $[28,36]$ in the same way that the BE FSS was tuned by piezoelectric-induced anisotropic strain [37]. We also note that the $|\widetilde{ \pm 1}\rangle$ with $|\widetilde{\mp 2}\rangle$ coupling $\Delta_{12}$ is a second-order term in VBM and should be weaker.

Estimation of the DB couplings $\Delta_{11}$ and $\Delta_{12}$ can be performed by assuming that the long-range contributions $\delta_{11}^{l r}$ and $\delta_{12}^{l r}$, proportional to $\delta_{0}^{l r}$, present the same dependence on the VBM parameters as the short-range parts. In this case, one can roughly replace $\delta_{0}^{s r}$ by $\Delta_{0}$, whose value can be found in the literature [16] and is close to $\Delta_{0} \sim 300 \mu \mathrm{eV}$. A statistical study of the VBM magnitude in Ref. [20] performed over 100 InGaAs/GaAs QDs from a sample similar to the one used in this work gives the mean values $\bar{\beta} \sim 0.2$ and $\bar{\gamma} \sim 0.1$. Using these values, we calculate that $\left|\Delta_{11}\right|$ is of the order of $35 \mu \mathrm{eV}$ and $\left|\Delta_{12}\right|$ is close to $5 \mu \mathrm{eV}$, taking the normalization parameters $\alpha_{e, l h}$ and $\alpha_{e, 2 l h}$ to be equal to 1 . We will show in the next section that the experimental determination of the DB couplings agrees well with this rough estimation.
TABLE I. Energy splittings of the excitonic level diagram. The results are given in series expansion where $\Delta_{0}$ is the dominant term. $\Delta_{D B}$ is defined as the splitting between the mean $\mathrm{BE}$ and $\mathrm{DE}$ energies.

\begin{tabular}{lcc}
\hline \hline QD symmetry & $C_{2 v}$ & $C_{s}$ \\
\hline FFS $_{B}$ & $\Delta_{1}$ & $\Delta_{1}-\frac{\Delta_{11} \Delta_{12}}{\Delta_{0}}$ \\
FFS $_{D}$ & $\Delta_{2}$ & $\Delta_{2}-\frac{\Delta_{11} \Delta_{12}}{\Delta_{0}}$ \\
$\Delta_{D B}$ & $\Delta_{0}$ & $\Delta_{0}+\frac{\Delta_{11} \Delta_{12}\left(2 \Delta_{0}^{2}+\Delta_{1} \Delta_{2}\right)}{2 \Delta_{0}^{3}}$ \\
\hline \hline
\end{tabular}

Our variational approach gives the same symmetry of the DB coupling terms as atomistic calculations of similarly shaped dots in Ref. [10]. Also, from a quantitative point of view, the numerical estimation of the DB mixing for a truncated QD shape given in Ref. [10] confirms that $\left|\Delta_{11}\right|>$ $\left|\Delta_{12}\right|$, which appears to be consistent with our model.

In addition, the symmetry reduction down to the $C_{s}$ symmetry leads to a renormalization of the excitonic energy levels, as depicted in Fig. 1(b). The FSS associated with the bright and dark states, named, respectively, $\mathrm{FSS}_{B}$ and $\mathrm{FSS}_{D}$, and the DB splitting $\Delta_{D B}$ depend on the $\Delta_{11}$ and $\Delta_{12}$ parameters. Table I gives the excitonic energy levels for both symmetries.

The additional contribution of the $C_{s}$ symmetry to the QD exchange splittings cannot be extracted easily, particularly when $\Delta_{11}$ and $\Delta_{12}$ remain weak compared to $\Delta_{0}$. In the following, we precisely present a method to measure the very weak DB mixing of a single InGaAs/GaAs QD using polarization-resolved photoluminescence experiments under magnetic field.

\section{EXPERIMENTAL RESULTS AND DISCUSSION}

Magnetospectroscopy of a single InGaAs QD was performed using a split-coil cryostat with optical access. A highnumerical-aperture (0.55) aspheric lens was used to focus the excitation beam from a He-Ne laser and to collect the PL. The positioning of the sample was ensured by Attocube piezostages. The sample was cooled down inside the pumped liquid-helium bath, and measurements were performed at $T=$ 1.5 K. The PL was dispersed using a 1-m focal length double spectrometer and was detected using a nitrogen-cooled CCD camera, providing a $15 \mu \mathrm{eV}$ spectral resolution.

Figure 2 shows the PL spectrum of the InGaAs/GaAs QD of interest at zero magnetic field. The BE and biexciton (XX) states were identified through their anticorrelated linearly polarized emission [see Fig. 2(a)] and the power-dependent evolution of the PL intensity, which showed linear and quadratic behaviors [see insets in Fig. 2(a)], respectively, for each component. The binding energy of the XX state $\Delta_{X X-B E}$, defined as $2 E_{B E}-E_{X X}$, is found here to be negative [38] since the XX line appears at higher energy than the BE.

The linear degree of polarization is a crucial parameter to be determined. Therefore, a careful measurement of the optical anisotropy of the BE is performed by rotating a $\lambda / 2$ plate 


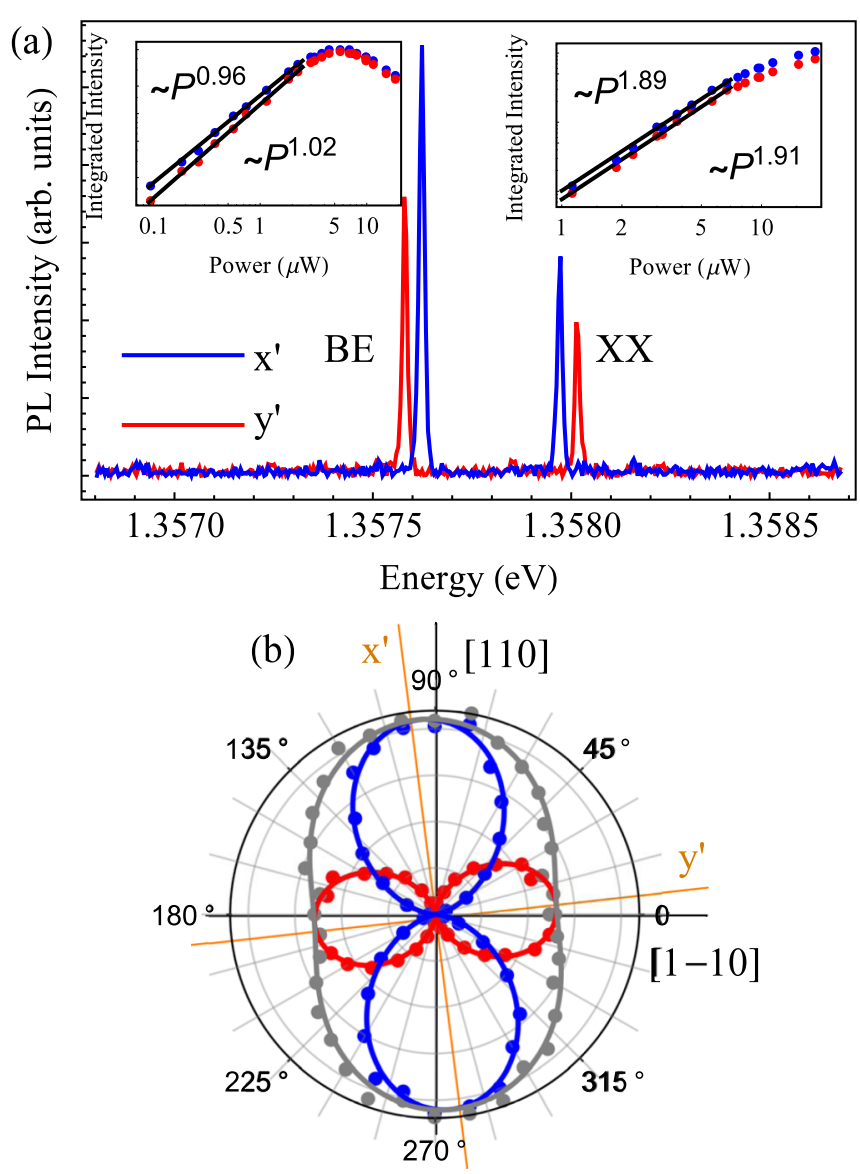

FIG. 2. (a) PL spectra recorded without applied magnetic field, showing the BE and XX states emission from the same QD. The linear polarization is analyzed along the two main QD axes $x^{\prime}$ and $y^{\prime}$. The insets show the integrated intensity of the BE and XX components as a function of the excitation power. (b) Polarization diagram of the two BE components. The gray dots correspond to the total intensity. The solid lines are fits using the model developed in Ref. [20].

in front of a fixed polarizer. The angular intensity distribution $I(\alpha)$ is then obtained, with $\alpha$ being the angle with respect to the [110] crystallographic axis. The angular dependences of both BE components are depicted in Fig. 2(b).

Analysis of the polar plot of the PL intensity of the BE lines [see Fig. 2(b)] clearly shows nonzero values of $\beta$ and $\theta$ since the maximum PL intensities of the two components have different magnitudes and are not aligned along the $\langle 110\rangle$ crystal directions. Based on previous work [20], we are able to extract the VBM parameter $\beta=|\beta| e^{2 i \psi}$ and the angle of the QD shape anisotropy orientation $\theta$. The values are gathered in Table II. The absence of emission from the DE state suggests that the DB coupling is much weaker than the DB splitting for this QD.

We performed magnetospectroscopy of the same QD using a tilted magnetic field configuration close to the Voigt geometry. The angle between the magnetic field and the sample plane was set to $\xi=3.5^{\circ}$, as shown in Fig. 3(a). The small perpendicular field component is required to obtain a change in the linear degree of polarization, while the in-plane component
TABLE II. Values of the different parameters responsible for the optical anisotropy determined by the analysis of the polarization diagram in Fig. 2(b). Values of the $g$ factors and exchange energies are deduced from the magnetospectroscopy experiment shown in Fig. 3(d). The error estimations are evaluated from the best fits.

\begin{tabular}{|c|c|}
\hline Parameter & Value \\
\hline \multicolumn{2}{|c|}{ Optical anisotropy } \\
\hline$\theta$ & $-3.7 \pm 0.2^{\circ}$ \\
\hline$|\beta|$ & $0.203 \pm 0.004$ \\
\hline $2 \psi$ & $-7.7 \pm 1^{\circ}$ \\
\hline \multicolumn{2}{|c|}{ Exchange parameters } \\
\hline$\Delta_{1}$ & $44.2 \pm 0.9 \mu \mathrm{eV}$ \\
\hline$\Delta_{2}$ & $-9.3 \pm 1.2 \mu \mathrm{eV}$ \\
\hline$\Delta_{D B}$ & $301.6 \pm 0.9 \mu \mathrm{eV}$ \\
\hline$\Delta_{11}$ & $-22 \pm 5 \mu \mathrm{eV}$ \\
\hline$\Delta_{12}$ & $18 \pm 5 \mu \mathrm{eV}$ \\
\hline \multicolumn{2}{|c|}{ Magnetic parameters } \\
\hline$g_{e}^{x}$ & $-0.356 \pm 0.008$ \\
\hline$g_{h}^{x}$ & $-0.642 \pm 0.008$ \\
\hline$g_{D}^{z}$ & $3.75 \pm 0.01$ \\
\hline$g_{B}^{z}$ & $2.907 \pm 0.005$ \\
\hline$\omega_{X}$ & $3.848 \pm 0.03 \mu \mathrm{eV} \mathrm{T}^{-2}$ \\
\hline \multicolumn{2}{|c|}{ Biexciton } \\
\hline$\Delta_{X X-B E}$ & $-693.8 \pm 0.9 \mu \mathrm{eV}$ \\
\hline$\omega_{X X}$ & $10.9 \pm 0.5 \mu \mathrm{eV} \mathrm{T}^{-2}$ \\
\hline
\end{tabular}

is needed to increase the DB mixing to make the DE optically active. The exciton transition energies are depicted in Fig. 3(c) as a function of the magnetic field. The two DE branches start appearing above $\sim 1.7 \mathrm{~T}$ due to the in-plane magnetic field component, which couples the BE and DE.

Simultaneously, on the high-energy side of the biexciton branches, additional lines appear with the same splitting. These high-energy lines are the optical transitions from the $\mathrm{XX}$ to the DE [labeled $|\mathrm{XX}\rangle \rightarrow\left|\mathrm{DE}_{H}\right\rangle$ and $|\mathrm{XX}\rangle \rightarrow\left|\mathrm{DE}_{L}\right\rangle$ in Fig. 3(b)].These transitions lie at higher energy than the $\mathrm{XX}$ emission since $\Delta_{X X-B E}$ is negative $[39,40]$. Their biexcitonic character is clearly identified through the superlinear behavior of the PL intensities as a function of the excitation power [see inset in Fig. 3(c)]. The full biexciton cascade in the presence of the magnetic field is depicted in Fig. 3(b), confirming the existence of eight lines. We take advantage of the extrabiexcitonlike branches to increase the accuracy with which the exchange and magnetic parameters are evaluated by fitting all the data shown in Fig. 3(d).

The diagonalization of the total Hamiltonian $\hat{H}_{\mathrm{exc}}+\hat{H}_{Z}$ gives the energy dependence on the magnetic field of the $\mathrm{BE}$ and DE branches. $\hat{H}_{\text {exc }}$ is taken, in a first approximation, as $\hat{H}_{C_{2 v}}$, and the Zeeman Hamiltonian $\hat{H}_{Z}$ is given in the exciton basis $|\widetilde{+1}\rangle,|\widetilde{-1}\rangle,|\widetilde{+2}\rangle,|\widetilde{\sim} 2\rangle$ by

$$
\frac{\mu_{B} B}{2}\left[\begin{array}{cccc}
g_{\mathrm{B}}^{z} \sin \xi & 0 & g_{e}^{x} \cos \xi & g_{h}^{x} \cos \xi \\
0 & -g_{\mathrm{B}}^{z} \sin \xi & g_{h}^{x} \cos \xi & g_{e}^{x} \cos \xi \\
g_{e}^{x} \cos \xi & g_{h}^{x} \cos \xi & g_{\mathrm{D}}^{z} \sin \xi & 0 \\
g_{h}^{x} \cos \xi & g_{e}^{x} \cos \xi & 0 & -g_{\mathrm{D}}^{z} \sin \xi
\end{array}\right],
$$

where $\mu_{B}$ is the Bohr magneton, $g_{e / h}^{x}$ are the in-plane Landé factors (the $x$ axis is taken along the [110] crystallographic 

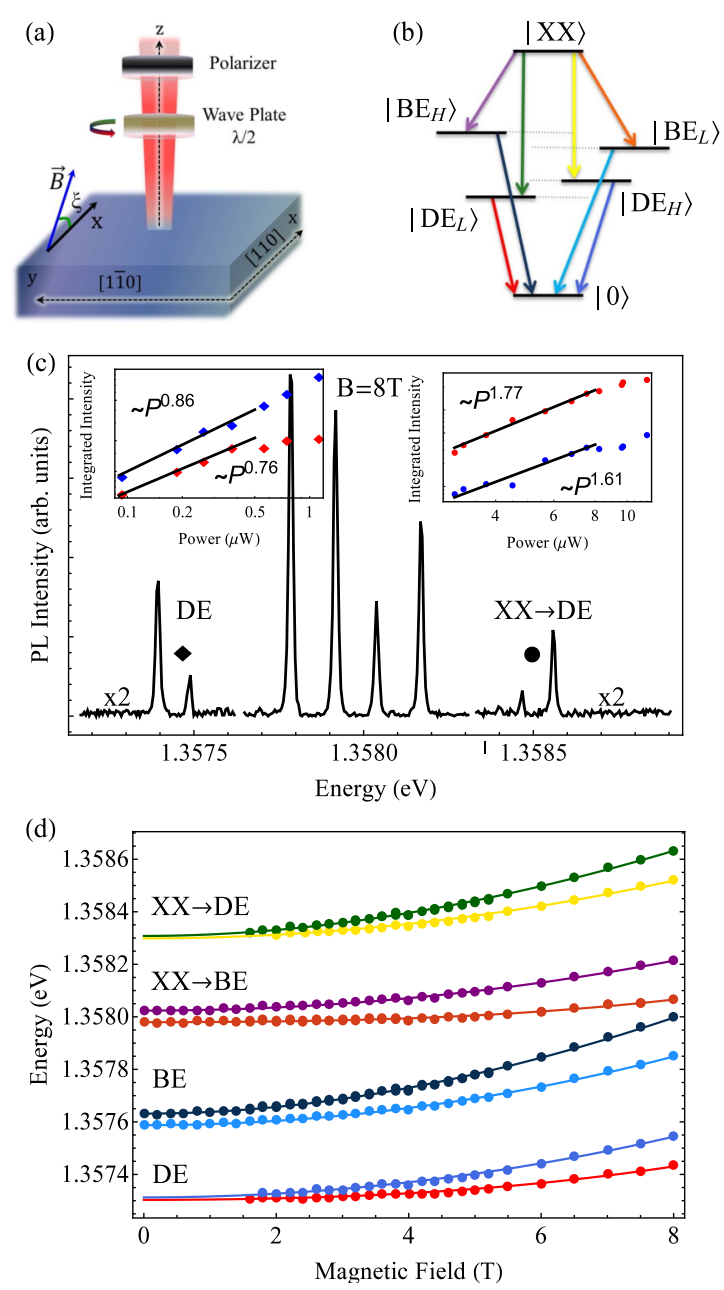

FIG. 3. (a) Sketch of the magnetic field orientation with respect to the sample plane. (b) Schematic of the full biexciton cascade involving the bright and dark excitons. (c) PL spectra for an applied magnetic field of $8 \mathrm{~T}$. The insets represent the power dependence of the dark exciton components and the two transitions from the biexciton to the dark exciton state, labeled $|\mathrm{XX}\rangle \rightarrow\left|\mathrm{DE}_{H}\right\rangle$ and $|\mathrm{XX}\rangle \rightarrow\left|\mathrm{DE}_{L}\right\rangle$. (d) Energies of the exciton and biexciton transitions versus magnetic field. The lines are fits using the $C_{2 v}$ electron/hole exchange Hamiltonian.

direction), and $g_{B}^{z}=g_{h}^{z}-g_{e}^{z}\left(g_{D}^{z}=g_{h}^{z}+g_{e}^{z}\right)$ is the longitudinal Landé factor for the BE (DE).

The four exciton branches are labeled by the eigenstates $\left\{\left|\mathrm{BE}_{H / L}\right\rangle,\left|\mathrm{DE}_{H / L}\right\rangle\right\}$, with the index $H / L$ referring to the high-/low-energy position. The diamagnetic contributions are taken into account and are given in Table II, where $\omega_{X}$ refers to the bright and dark excitons and $\omega_{X X}$ refers to the biexciton diamagnetic shift. The $|\mathrm{XX}\rangle \rightarrow|\mathrm{BE}\rangle(|\mathrm{XX}\rangle \rightarrow|\mathrm{DE}\rangle)$ transitions are calculated by expressing the energy difference between the spin $S=0$ of the $|\mathrm{XX}\rangle$ state, which is affected by only the diamagnetic shift, and the BE (DE) state, calculated using the $C_{2 v}$ approximation [16].

Polarization maps as shown in Fig. 2(b) were carried out at different magnetic fields to quantify the degree of linear polarization of each transition $\rho_{L}$, defined as $\left(I_{x^{\prime}}-I_{y^{\prime}}\right) /\left(I_{x^{\prime}}+\right.$ $\left.I_{y^{\prime}}\right)$. Here, $x^{\prime}$ is the maximum intensity axis, and $y^{\prime}$ is the minimum, with $x^{\prime}$ and $y^{\prime}$ being orthogonal [41].
Figure 4 shows $\rho_{L}$ as a function of the applied magnetic field for BEs and DEs. The DE data are shown only over the range [3-8 T] since the DE PL intensity between $1.7 \mathrm{~T}$ (where the DE becomes visible) and $3 \mathrm{~T}$ was not sufficient to allow an accurate evaluation of $\rho_{L}$. It can be seen from Figs. 4(c) and 4(d) that $\rho_{L}$ decreases slightly (from $100 \%$ at 0 $\mathrm{T}$ to $90 \%-95 \%$ at higher fields) for the BE branches. This is due to the presence of the small longitudinal component of the applied magnetic field [42] [Fig. 3(a)]. However, the two DE branches show remarkably different dependences on the applied magnetic field [Figs. 4(a) and 4(b)]. For the $\left|\mathrm{DE}_{L}\right\rangle$ line, $\rho_{L}$ is almost $100 \%$, with no strong dependence on magnetic field, whereas for the $\left|\mathrm{DE}_{H}\right\rangle$ line, $\rho_{L}$ decreases markedly from around $80 \%$ to $20 \%$ over the $[3-8 \mathrm{~T}]$ interval. This decrease in $\rho_{L}$ depends on the angle of the tilted magnetic field $\xi$, with a greater rate of change in $\rho_{L}$ for higher values of $\xi$. Since $\rho_{L}$ depends on both the longitudinal and transverse components of the magnetic field in a nontrivial way, the rate of variation of $\rho_{L}$ with magnetic field can be used as a sensitive probe to evaluate the amplitude of $\Delta_{11}$ and $\Delta_{12}$. This can be understood by the fact that although the reduction in $\rho_{L}$ is due to the applied perpendicular magnetic field, the effective in-plane magnetic field acting on the exciton is modified by DB couplings, as described by the $C_{s}$ Hamiltonian shown earlier.

The degree of linear polarization can be calculated through the following expression:

$$
\rho_{L}(\phi)=\frac{\sqrt{\left(\left|\widetilde{P}_{x^{\prime}}^{\phi}\right|^{2}-\left|\widetilde{P}_{y^{\prime}}^{\phi}\right|^{2}\right)^{2}+4\left(\operatorname{Re}\left[\widetilde{P}_{x^{\prime}}^{\phi} \widetilde{P}_{y^{\prime}}^{\phi, *}\right]\right)^{2}}}{\left|\widetilde{P}_{x^{\prime}}^{\phi}\right|^{2}+\left|\widetilde{P}_{y^{\prime}}^{\phi}\right|^{2}},
$$

where $\widetilde{P}_{x^{\prime}}^{\phi}=\left\langle 0\left|\hat{p}_{x^{\prime}}\right| \phi\right\rangle$ and $\widetilde{P}_{y^{\prime}}^{\phi}=\left\langle 0\left|\hat{p}_{y^{\prime}}\right| \phi\right\rangle$ are related to the optical matrix elements. Here, $\hat{p}_{i}$ are the momentum operators, and $|\phi\rangle=\left\{\left|\mathrm{BE}_{H / L}\right\rangle,\left|\mathrm{DE}_{H / L}\right\rangle\right\}$. These states are evaluated for a given magnetic field, and the effect of VBM on polarization properties is taken into consideration as $|\phi\rangle=$ $\sum_{i} c_{i}^{\phi}(B)|\widetilde{i}\rangle$, with $i=\{1,-1,+2,-2\}$. The polarizations of the DE states are fully determined by their bright components $c_{ \pm 1}^{D E_{H}}$ and $c_{ \pm 1}^{D E_{L}}$ since $\left\langle 0\left|\hat{p}_{x / y}\right| \widetilde{ \pm 2}\right\rangle$ is equal to zero. The evolution with magnetic field of $\rho_{L}$ for the transitions $|\mathrm{XX}\rangle \rightarrow\left|\mathrm{BE}_{H / L}\right\rangle\left(|\mathrm{XX}\rangle \rightarrow\left|\mathrm{DE}_{H / L}\right\rangle\right)$ was observed experimentally to have the same magnetic field dependence as the $\left|\mathrm{BE}_{L / H}\right\rangle\left(\left|\mathrm{DE}_{L / H}\right\rangle\right)$ branches as a consequence of the angular momentum conservation in the $\mathrm{XX}$ radiative cascade.

The simulation of the $\rho_{L}$ evolution with applied magnetic field using the approximation $\hat{H}_{\mathrm{exc}}=\hat{H}_{C_{2 v}}$ to calculate $\widetilde{P}_{x}^{\phi}$ and $\widetilde{P}_{y}^{\phi}$ is given by the blue curve in Fig. 4 . Note that only the parameters gathered in Table II are required to give a fit to the experimental results for the two BEs and the lowerenergy DE.

However, the $\rho_{L}$ dependence calculated using the $C_{2 v}$ Hamiltonian predicts a much lower degree of linear polarization in the $\mathrm{DE}_{H}$ branch than observed experimentally. This discrepancy between calculation and experimental data when using the $C_{2 v}$ Hamiltonian demonstrates the importance of the DB coupling induced by breaking of the QD symmetry, as described by parameters $\Delta_{11}$ and $\Delta_{12}$. When this asymmetry is taken into account (calculation with $C_{s}$ symmetry, shown by the red curve in Fig. 4), the degree of linear polarization is higher and agrees better with the experimental data. This 


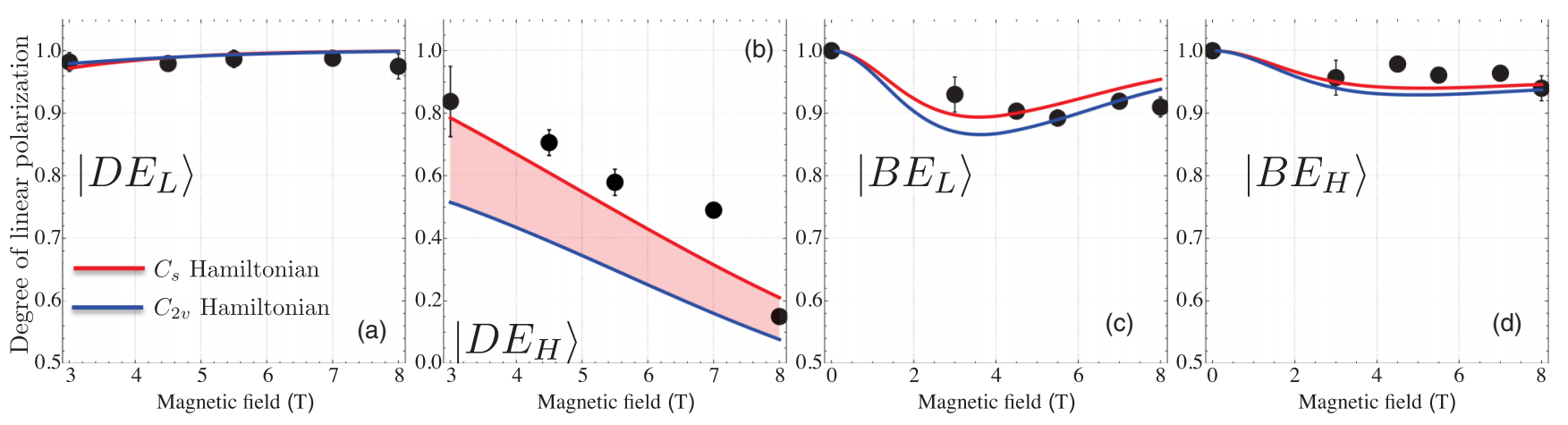

FIG. 4. Evolution of the degree of linear polarization as a function of the applied magnetic field for the (a) and (b) dark and (c) and (d) bright exciton branches. The fit residual values do not exceed $4 \%$ for the whole range of magnetic field in (a), (c), and (d). This stresses the good quality of the fit, despite unavoidable experimental noise.

indicates that the effect of dot asymmetry is to increase the effective in-plane magnetic field component seen by the $\mathrm{DE}_{H}$ through the action of $\Delta_{11}$ and $\Delta_{12}$ on the exciton mixing. This effect is evident only in the high-energy DE branch due to its closer proximity to the $\mathrm{BE}$, which leads to a high sensitivity to DB mixing parameters; the lower-energy DE branch is well separated energetically from the BE and hence shows no such sensitivity.

The red curves in Fig. 4 show the effect of including the DB coupling terms $\Delta_{11}$ and $\Delta_{12}$ in the calculation of $\rho_{L}(\phi)$. This was achieved by using the full-exchange Hamiltonian $\hat{H}_{\text {exc }}$ to numerically calculate the new $\left\{c_{i}^{\phi}\right\}$ weights. We restrict the fitting procedure to real values, and the four sets of data were treated simultaneously, resulting in a fit with a high degree of sensitivity to the values of $\Delta_{11}$ and $\Delta_{12}$. The result is presented in Fig. 4 by red curves, showing very good agreement between calculated and experimental data for the $\left|\mathrm{DE}_{H}\right\rangle$ branch in particular. We find that $\Delta_{11}=-22 \mu \mathrm{eV}$ and $\Delta_{12}=18 \mu \mathrm{eV}$ (also reported in Table II), values that are close to those estimated in Sec. II. Note that the signs of the couplings contribute to the increase in the in-plane magnetic field components, as previously assumed. The perturbation induced by the DB mixing parameters $\Delta_{11}$ and $\Delta_{12}$ is quite weak for the bright branches. However, an increase of the $\Delta_{11}$ and $\Delta_{12}$ amplitudes by more than $5 \mu \mathrm{eV}$ results in a large deviation in the calculated value of the $\mathrm{DE}_{L}$ polarization from the experimental values. This allows the determination of the error in $\Delta_{11}$ and $\Delta_{12}$ to be within $\pm 5 \mu \mathrm{eV}$ when fitting all four data sets simultaneously.

Once $\Delta_{11}$ and $\Delta_{12}$ are known, the oscillator strength $f_{\phi}$ of the four exciton states can be evaluated at zero magnetic field. We find then $\left\{f_{B E_{H}}, f_{B E_{L}}, f_{D E_{H}}, f_{D E_{L}}\right\}=$ $\left\{1,0.62,3.8 \times 10^{-3}, 1.1 \times 10^{-4}\right\}$, which is in agreement with the experimental absence of the DE luminescence at zero field, for the dot shown in Fig. 1(a). We can note that the $\Delta_{11}$ and $\Delta_{12}$ couplings play no significant role in the $\mathrm{BE}$ oscillator strength ratio, which is mainly governed by the VBM amplitude $\beta$ [43].

As already mentioned in the Introduction, these DB coupling terms are responsible for the anticrossing between the $\mathrm{DE}_{H}$ and $\mathrm{BE}_{L}$ branches in the Faraday configuration. Using the parameters in Table II, we expect that a very weak anticrossing will occur at $1.8 \mathrm{~T}$ with a crossing amplitude of $14 \mu \mathrm{eV}$ and an avoided-crossing region spreading over $5 \times 10^{-2} \mathrm{~T}$, making it extremely difficult to detect even with $15 \mu \mathrm{eV}$ spectral resolution.

The estimation of $\Delta_{11}$ and $\Delta_{12}$ based on the analysis of the degree of polarization of the DE presented here relies on the assumption that the parameters $\Delta_{11}$ and $\Delta_{12}$ are weak enough that the exchange and magnetic QD parameters, obtained in the framework of the $C_{2 v}$ approximation, are not altered. This assumption is validated by including the DB couplings in the calculation of the dependence of the DE and BE emission energy with magnetic field. The $\Delta_{11}$ and $\Delta_{12}$ energies lead to a weak renormalization of the DB splitting $\Delta_{D B}$, FSS of the bright exciton $\left(\mathrm{FSS}_{B}\right)$ and FSS of the dark exciton $\left(\mathrm{FSS}_{D}\right)$ by less than $\sim 1 \mu \mathrm{eV}$ (see Table I). Consequently, the Landé factors are not modified. Note that this conclusion is valid for QDs for which the condition $\left|\Delta_{11}\right|,\left|\Delta_{12}\right| \ll \Delta_{D B}$ is fulfilled.

\section{CONCLUSION}

We have implemented an experimental method to measure weak DB exciton mixing. It is based on a detailed analysis of the linear polarization of the exciton emission in a Voigt magnetic field with a small additional longitudinal magnetic field component. This method involves recording the polarization diagram of $\mathrm{DE}$ and $\mathrm{BE}$ as a function of applied magnetic field, resulting in an accurate procedure to determine $\Delta_{11}$ and $\Delta_{12}$. Although it is expected that the magnitude of $\Delta_{11}$ and $\Delta_{12}$ is linked to the $\gamma$ VBM parameter strength, this correlation has not been clearly demonstrated experimentally. Therefore, the advantage of the method shown here is that it relies solely on the determination of the $\beta$ VBM parameter, which can be found by a simple analysis of the emission polarization along the growth direction. This study can contribute to improving the control of the coupling between $\mathrm{DE}$ and $\mathrm{BE}$ by, for example, vibrational strain [36] selecting QDs with a native DB mixing, making long-lived coherent states available for quantum optics.

\section{ACKNOWLEDGMENTS}

This work was financially supported by the French National Research Agency (ANR "SPINEX" ANR-17-CE300022-01) and by the Cluster of Excellence MATISSE led by 
Sorbonne Universités. This work was partly supported by the French RENATECH network.

\section{APPENDIX A}

The hole Hamiltonian reads $\hat{H}_{h}=\hat{H}_{k}+\hat{H}_{\epsilon}+V_{Q D} \hat{I}$, composed of the kinetic Hamiltonian, the strain Hamiltonian, and the heterostructure potential, including the hydrostatic part of the strain, respectively. In the $(+3 / 2,+1 / 2,-1 / 2,-3 / 2)$ basis, $\hat{H}_{k}$ reads

$$
\left[\begin{array}{cccc}
P_{k}+Q_{k} & -S_{k} & R_{k} & 0 \\
-S_{k}^{*} & P_{k}-Q_{k} & 0 & R_{k}^{*} \\
R_{k}^{*} & 0 & P_{k}-Q_{k} & S_{k} \\
0 & R_{k}^{*} & S_{k}^{*} & P_{k}+Q_{k}
\end{array}\right],
$$

where $P_{k}=\frac{\hbar^{2} \gamma_{1}}{2 m_{0}}\left(k_{x}^{2}+k_{y}^{2}+k_{z}^{2}\right), Q_{k}=\frac{\hbar^{2} \gamma_{1}}{2 m_{0}}\left(k_{x}^{2}+k_{y}^{2}-2 k_{z}^{2}\right)$, $R_{k}=\frac{\hbar^{2}}{2 m_{0}}\left[-\sqrt{3}\left(k_{x}^{2}-k_{y}^{2}\right)+2 i \sqrt{3} \gamma_{2} k_{x} k_{y}\right], \quad$ and $\quad S_{k}=\frac{\hbar^{2} \gamma_{3}}{2 m_{0}}$ $\sqrt{3}\left(k_{x}-i k_{y}\right) k_{z}$. Here, $k_{i}$ are the differential operators $-i \frac{\partial}{\partial x_{i}}$. The strain Hamiltonian $\hat{H}_{\epsilon}$ is obtained by applying the following replacement rules: $\left(P_{k}, Q_{k}, R_{k}, S_{k}\right) \rightarrow$ $\left(P_{\epsilon}, Q_{\epsilon}, R_{\epsilon}, S_{\epsilon}\right)$ with $k_{i} k_{j} \rightarrow \epsilon_{i j}$ and $\left(\frac{\hbar^{2} \gamma_{1}}{2 m_{0}}, \frac{\hbar^{2} \gamma_{2}}{2 m_{0}}, \frac{\hbar^{2} \gamma_{3}}{2 m_{0}}\right) \rightarrow$ $\left(-a_{v},-b / 2,-d / 2 \sqrt{3}\right)$. The $\left\{\gamma_{i}\right\}$ and $\left\{a_{v}, b, d\right\}$ are the Luttinger parameters and strain elastic coefficients for InGaAs. We also define the $\mathrm{HH}$ to $\mathrm{LH}$ energy splitting $\Delta_{L H}=\left\langle\chi_{h h}\left|P_{k}+P_{\epsilon}+Q_{k}+Q_{\epsilon}+V_{Q D}\right| \chi_{h h}\right\rangle-\left\langle\chi_{l h}\right| P_{k}+$ $P_{\epsilon}-Q_{k}-Q_{\epsilon}+V_{Q D}\left|\chi_{l h}\right\rangle$, where $\chi_{h h / l h}$ are the envelope wave functions associated with the $\mathrm{HH} / \mathrm{LH}$ states calculated in the absence of the off-diagonal terms in $\hat{H}_{h}$.

\section{APPENDIX B}

We show here the normalization integrals present in the short-range contributions:

$$
\begin{aligned}
\alpha_{e, l h} & =\frac{\int \chi_{e}^{2}(\mathbf{r}) \chi_{h h}(\mathbf{r}) \chi_{l h}(\mathbf{r}) d^{3} \mathbf{r}}{\int \chi_{e}^{2}(\mathbf{r}) \chi_{h h}^{2}(\mathbf{r}) d^{3} \mathbf{r}}, \\
\alpha_{e, 2 l h} & =\frac{\int \chi_{e}^{2}(\mathbf{r}) \chi_{l h}^{2}(\mathbf{r}) d^{3} \mathbf{r}}{\int \chi_{e}^{2}(\mathbf{r}) \chi_{h h}^{2}(\mathbf{r}) d^{3} \mathbf{r}} .
\end{aligned}
$$

[1] A. Dousse, J. Suffczyński, A. Beveratos, O. Krebs, A. Lemaître, I. Sagnes, J. Bloch, P. Voisin, and P. Senellart, Nature (London) 466, 217 (2010).

[2] D. Press, K. De Greve, P. L. McMahon, T. D. Ladd, B. Friess, C. Schneider, M. Kamp, S. Höfling, A. Forchel, and Y. Yamamoto, Nat. Photonics 4, 367 (2010).

[3] Y. He, Y.-M. He, Y.-J. Wei, X. Jiang, K. Chen, C.-Y. Lu, J.-W. Pan, C. Schneider, M. Kamp, and S. Höfling, Phys. Rev. Lett. 119, 060501 (2017).

[4] A. Reigue, J. Iles-Smith, F. Lux, L. Monniello, M. Bernard, F. Margaillan, A. Lemaitre, A. Martinez, D. P. S. McCutcheon, J. Mørk, R. Hostein, and V. Voliotis, Phys. Rev. Lett. 118, 233602 (2017)

[5] B. Siarry, B. Eble, F. Bernardot, P. Grinberg, C. Testelin, M. Chamarro, and A. Lemaître, Phys. Rev. B 92, 155315 (2015).

[6] E. Poem, Y. Kodriano, C. Tradonsky, N. H. Lindner, B. D. Gerardot, P. M. Petroff, and D. Gershoni, Nat. Phys. 6, 993 (2010).

[7] I. Schwartz, E. R. Schmidgall, L. Gantz, D. Cogan, E. Bordo, Y. Don, M. Zielinski, and D. Gershoni, Phys. Rev. X 5, 011009 (2015).

[8] J. Puebla, E. A. Chekhovich, M. Hopkinson, P. Senellart, A. Lemaitre, M. S. Skolnick, and A. I. Tartakovskii, Phys. Rev. B 88, 045306 (2013).

[9] T. Smoleński, T. Kazimierczuk, M. Goryca, P. Wojnar, and P. Kossacki, Phys. Rev. B 91, 155430 (2015).

[10] M. Zieliński, Y. Don, and D. Gershoni, Phys. Rev. B 91, 085403 (2015).

[11] F. Fras, B. Eble, F. Bernardot, C. Testelin, M. Chamarro, A. Miard, and A. Lemaître, Appl. Phys. Lett. 100, 012104 (2012).

[12] S. Bounouar, C. Morchutt, M. Elouneg-Jamroz, L. Besombes, R. André, E. Bellet-Amalric, C. Bougerol, M. Den Hertog, K. Kheng, S. Tatarenko, and J. Ph. Poizat, Phys. Rev. B 85, 035428 (2012).
[13] S. Sancho, M. Chaouache, M. A. Maaref, F. Bernardot, B. Eble, A. Lemaître, and C. Testelin, Phys. Rev. B 84, 155458 (2011).

[14] K. Peng, S. Wu, J. Tang, F. Song, C. Qian, S. Sun, S. Xiao, M. Wang, H. Ali, D. A. Williams, and X. Xu, Phys. Rev. Appl. 8, 064018 (2017).

[15] A. Lafuente-Sampietro, H. Utsumi, H. Boukari, S. Kuroda, and L. Besombes, Phys. Rev. B 93, 161301 (2016).

[16] M. Bayer, G. Ortner, O. Stern, A. Kuther, A. A. Gorbunov, A. Forchel, P. Hawrylak, S. Fafard, K. Hinzer, T. L. Reinecke, S. N. Walck, J. P. Reithmaier, F. Klopf, and F. Schäfer, Phys. Rev. B 65, 195315 (2002).

[17] K. F. Karlsson, M. A. Dupertuis, D. Y. Oberli, E. Pelucchi, A. Rudra, P. O. Holtz, and E. Kapon, Phys. Rev. B 81, 161307 (2010).

[18] M. A. Dupertuis, K. F. Karlsson, D. Y. Oberli, E. Pelucchi, A. Rudra, P. O. Holtz, and E. Kapon, Phys. Rev. Lett. 107, 127403 (2011).

[19] J.-W. Luo, G. Bester, and A. Zunger, Phys. Rev. B 92, 165301 (2015).

[20] C. Tonin, R. Hostein, V. Voliotis, R. Grousson, A. Lemaitre, and A. Martinez, Phys. Rev. B 85, 155303 (2012).

[21] T. Belhadj, T. Amand, A. Kunold, C. M. Simon, T. Kuroda, M. Abbarchi, T. Mano, K. Sakoda, S. Kunz, X. Marie, and B. Urbaszek, Appl. Phys. Lett. 97, 051111 (2010).

[22] S. Ohno, S. Adachi, R. Kaji, S. Muto, and H. Sasakura, Appl. Phys. Lett. 98, 161912 (2011).

[23] T. Smoleński, T. Kazimierczuk, M. Goryca, T. Jakubczyk, Ł. Kłopotowski, Ł. Cywiński, P. Wojnar, A. Golnik, and P. Kossacki, Phys. Rev. B 86, 241305 (2012).

[24] Y. H. Huo, V. Křápek, O. G. Schmidt, and A. Rastelli, Phys. Rev. B 95, 165304 (2017).

[25] D. N. Krizhanovskii, A. Ebbens, A. I. Tartakovskii, F. Pulizzi, T. Wright, M. S. Skolnick, and M. Hopkinson, Phys. Rev. B 72, 161312 (2005). 
[26] S. Lee, O. L. Lazarenkova, P. von Allmen, F. Oyafuso, and G. Klimeck, Phys. Rev. B 70, 125307 (2004).

[27] The geometry of the potential fully governs the symmetry of the wave functions when a homogenous QD composition is assumed; otherwise, it leads to increasing $|\gamma|$.

[28] E. Tsitsishvili, Phys. E 87, 161 (2017).

[29] C.-H. Lin, W.-T. You, H.-Y. Chou, S.-J. Cheng, S.D. Lin, and W.-H. Chang, Phys. Rev. B 83, 075317 (2011).

[30] E. L. Ivchenko and G. E. Pikus, Superlattices and Other Heterostructures, Springer Series in Solid-State Sciences, Vol. 110 (Springer, Berlin, 1997).

[31] Y. Léger, L. Besombes, L. Maingault, and H. Mariette, Phys. Rev. B 76, 045331 (2007).

[32] M. Gawełczyk, M. Syperek, A. Maryński, P. Mrowiński, L. Dusanowski, K. Gawarecki, J. Misiewicz, A. Somers, J. P. Reithmaier, S. Höfling, and G. Sęk, Phys. Rev. B 96, 245425 (2017).

[33] K. Kowalik, O. Krebs, A. Lemaître, J. A. Gaj, and P. Voisin, Phys. Rev. B 77, 161305 (2008).

[34] T. Takagahara, Phys. Rev. B 62, 16840 (2000).
[35] H. Tong and M. W. Wu, Phys. Rev. B 83, 235323 (2011).

[36] R. Ohta, H. Okamoto, T. Tawara, H. Gotoh, and H. Yamaguchi, Phys. Rev. Lett. 120, 267401 (2018).

[37] J. D. Plumhof, R. Trotta, V. Křápek, E. Zallo, P. Atkinson, S. Kumar, A. Rastelli, and O. G. Schmidt, Phys. Rev. B 87, 075311 (2013).

[38] R. J. Young, R. M. Stevenson, A. J. Shields, P. Atkinson, K. Cooper, D. A. Ritchie, K. M. Groom, A. I. Tartakovskii, and M. S. Skolnick, Phys. Rev. B 72, 113305 (2005).

[39] B. J. Witek, R. W. Heeres, U. Perinetti, E. P. A. M. Bakkers, L. P. Kouwenhoven, and V. Zwiller, Phys. Rev. B 84, 195305 (2011).

[40] L. Besombes, K. Kheng, and D. Martrou, Phys. Rev. Lett. 85, 425 (2000).

[41] Note that the direction of $x^{\prime}$ and $y^{\prime}$ attached to a particular BE component rotates by increasing the magnetic field magnitude as a consequence of the finite value $\theta$.

[42] M. Paillard, X. Marie, P. Renucci, T. Amand, A. Jbeli, and J. M. Gerard, Phys. Rev. Lett. 86, 1634 (2001).

[43] L.-W. Wang, J. Kim, and A. Zunger, Phys. Rev. B 59, 5678 (1999). 